\title{
De la guerrière à la citoyenne. Porter les armes pendant l'Ancien Régime et la Révolution française
}

Dominique Godineau

\section{(2) OpenEdition}

1 Journals

Édition électronique

URL : https://journals.openedition.org/clio/1418

DOI : $10.4000 /$ clio. 1418

ISSN : 1777-5299

Éditeur

Belin

Édition imprimée

Date de publication : 1 novembre 2004

ISBN : 2-85816-755-9

ISSN : $1252-7017$

Référence électronique

Dominique Godineau, « De la guerrière à la citoyenne. Porter les armes pendant l'Ancien Régime et la Révolution française », Clio. Histoire, femmes et sociétés [En ligne], 20 | 2004, mis en ligne le 23 août 2013, consulté le 22 avril 2022. URL : http://journals.openedition.org/clio/1418 ; DOI : https://doi.org/ 10.4000/clio.1418

Ce document a été généré automatiquement le 22 avril 2022

Tous droits réservés 


\title{
De la guerrière à la citoyenne. Porter les armes pendant l'Ancien Régime et la Révolution française
}

\author{
Dominique Godineau
}

1 À l'époque moderne, quelques femmes en France ont porté les armes, guerroyé ou servi dans les armées du Roi, puis de la République. Elles représentent une proportion tout à fait infime des soldats, et leur exhumation des archives ne bouleverse certes pas l'histoire des institutions militaires. En termes quantitatifs, on est là sur les marges, à la limite de l'anecdotique. On peut toutefois dépasser l'anecdote pour s'interroger, à partir de ces marges, sur les rapports entre armes, guerre, masculin, féminin. Les histoires concrètes de ces femmes soldats, leurs propos et ceux tenus sur elles aident à comprendre les mécanismes de l'exclusion des femmes des armées. Renouant avec la conception du citoyen armé et l'image du soldat-citoyen, la Révolution introduit une autre dimension, politique, dans ces rapports complexes. En ce sens, elle marque un tournant et conduit à enrichir le questionnement : de quoi est faite l'association entre masculin, armes et citoyenneté ? Que signifie, pour une femme pendant la Révolution, demander le port des armes ou combattre comme soldat? Avant d'examiner ces points, il convient d'abord de présenter la situation pendant l'Ancien Régime, dans une perspective comparatiste permettant de mesurer le degré de la rupture révolutionnaire.

\section{Nobles guerrières et soldates roturières}

Dans la société d'Ancien Régime, guerre et port des armes sont liés à deux qualités : la noblesse et le masculin. Certes, tous les soldats ne sont évidemment pas nobles et tous les nobles ne sont pas soldats, loin s'en faut. Mais, selon l'historique tripartition de la société, le second ordre du royaume reste défini par sa fonction combattante et ses qualités guerrières - ce que rappellent les titres d'écuyer et de chevalier ou le privilège du port de l'épée. Jusqu'à la moitié du XVII siècle, la plupart des grands nobles sont des 
guerriers, élevés pour combattre, faisant la guerre à la tête de leurs troupes, tirant prestige de leur bravoure et de leurs victoires. Chef militaire du royaume, le monarque est lui-même un « roi de guerre » assumant le commandement réel des troupes.

Valorisée dans la société aristocratique, la chose guerrière est également associée au masculin. Là encore, si tous les hommes ne sont pas des soldats, le masculin est, aux $\mathrm{XVI}^{\mathrm{e}}-\mathrm{XVII}{ }^{e}$ siècles, symbolisé en grande partie par la guerre, le combat, les armes, en opposition au féminin. Image du monde à l'envers, une gravure du XVII ${ }^{e}$ siècle ${ }^{1}$ représente ainsi un homme assis, un nourrisson sur ses genoux, une coiffe sur la tête et tenant une quenouille, tandis que, debout près de la porte (l'extérieur), dans une position dominante, se dresse devant lui sa femme casquée, épée au côté, mousquet sur l'épaule, main levée et doigt pointé vers lui en signe de commandement. Ayant vécu les tumultes de la Guerre de Trente Ans et de la Fronde, $\mathrm{M}^{\mathrm{me}}$ de la Guette, noble briarde, assure dans ses Mémoires se sentir plus proche du masculin que du féminin car elle préfère la guerre aux paisibles occupations domestiques des femmes. Dans un portrait rimé d'elle-même, elle écrit ainsi :

Je sais fort bien que ma démarche

Tient un peu trop du masculin ;

Mais je dis que le féminin

Ne fut jamais ce qui m'attache.

Si je suivais ma fantaisie,

Je m'en irais dans les combats,

Avec un fort grand coutelas,

Faire une étrange boucherie.

Elle avait déjà prévenu le lecteur: "J'ai toujours été d'une humeur plus portée à la guerre qu'aux exercices tranquilles de mettre les poules à couver et de filer la quenouille, quoique l'on dise qu'une femme ne doit savoir que cela.» Et elle évoque plusieurs fois le " grand plaisir » qu'elle prend à parler de la guerre ou à entendre les roulements des tambours, les fanfares des trompettes et du canon, "plus agréables que les meilleurs symphonies $»^{2}$. Excellente cavalière, elle est également habile au pistolet, au fleuret et au fusil, ayant obtenu dans sa jeunesse que son père (qui n'avait pas de fils) lui donne un maître d'armes, chose peu habituelle pour une fille mais qui n'a cependant rien de scandaleux. Épouse et mère aimante et respectable, $\mathrm{M}^{\mathrm{me}}$ de la Guette ne transgresse d'ailleurs pas son rôle et reste dans la norme. Insister, comme elle le fait dans ses Mémoires, sur son caractère guerrier et masculin est un moyen de se distinguer, de se mettre en valeur et finalement de faire ressortir sa noblesse.

5 Si elle n'a pas combattu, d'autres nobles ont été amenées à le faire dans les temps troublés des guerres de religion et de la Fronde. Éliane Viennot et Sylvie Steinberg ${ }^{3}$ en ont repéré une quinzaine, citées par les mémorialistes, chroniqueurs et historiens de l'époque. Parmi elles, des princesses passant les troupes en revue comme Catherine de Médicis qui, «s'exposant aux arquebuses et canonnades comme un de ses capitaines " (Brantôme), conduisit l'armée française au siège du Havre, ou comme $\mathrm{M}^{\text {lle }}$ de Montpensier devant Orléans en 1652. Trois mois plus tard, cette dernière faisait tirer le canon de la Bastille sur l'armée royale, sauvant les Frondeurs. Plus fréquemment, c'était pour défendre leur domaine assiégé que, en l'absence de leur mari, des femmes nobles dirigeaient la résistance face à l'ennemi, parfois les armes à la main. En 1590, l'épouse de Coligny, à la tête de ses serviteurs, mit par exemple en fuite les Ligueurs à Châtillon-sur-Loing. Ces femmes étaient souvent veuves, telle la protestante limousine Madeleine de Miraumont qui, selon Agrippa d'Aubigné (Histoire universelle, 1618), avait 
"dressé une compagnie de cavalerie de soixante gentilshommes » qu'elle menait à la bataille, « connue par amis et ennemis à ses cheveux qui dessous la salade [le casque] lui couvraient l'échine ». La plus célèbre de ces guerrières est la Lorraine Saint-Balmont qui, pendant la Fronde, épée ou pistolet à la main, bottée et portant l'habit d'homme, défendait ses terres pendant que son époux faisait campagne; d'après son biographe, c'était celui-ci qui l'avait entraînée à " tous les exercices de la guerre et des armes » et lui donnait des leçons d'équitation ${ }^{4}$.

Dans cette société encore fortement structurée par les liens familiaux, il était du devoir des femmes de remplacer un mari pour gérer, et au besoin défendre, le patrimoine familial. Cela faisait partie de leur rôle, mais n'était tout de même pas courant : c'est bien parce que leur conduite guerrière était peu commune pour une femme que certaines sont devenues célèbres. De plus, l'on n'était jamais loin de la transgression, car une femme se battant ou se conduisant comme un homme brouillait les frontières de genre. $\mathrm{M}^{\mathrm{me}}$ de la Guette raconte ainsi que ses manières, ses goûts, sa conversation étonnaient ceux qu'elle rencontrait, au point que certains l'auraient même prise pour un gentilhomme déguisé en femme. Les exploits militaires féminins suscitaient d'ailleurs à la fois fascination devant la «merveille $»^{5}$ et réserves face à la transgression.

Que les femmes fassent les femmes, non les capitaines : si la maladie de leurs maris, la minorité de leurs enfants, les contraignent se présenter au combat, cela est tolérable pour une fois ou deux en la nécessité; il leur est plus séant se mêler des affaires en une bonne ville proche des armées, que d'entrer en icelle, où elles sont injuriées des ennemis et moquées des amis'.

7 Tavannes pose clairement les limites: en cas de "nécessité », les dames peuvent remplacer les hommes, ou participer à la protection d'une ville assiégée en réparant les murailles, en encourageant les combattants, voire en prenant les armes (Poitiers 1569, La Rochelle 1572). Mais leur place n'est pas sur les champs de bataille, car il n'est guère bienséant de voir une femme, armée et vêtue comme un homme, se distinguer par des exploits individuels qui vont à l'encontre des qualités féminines de réserve et modestie - sans compter que la présence des soldats fait toujours planer le danger de viol. Et surtout, dans tous les cas, leur combat doit être défensif et non conquérant. De fait, ces nobles héroïnes se sont battues pour défendre leur domaine, et non pour aller porter le fer à l'étranger; et c'est d'ailleurs souvent parce que leur mari guerroyait au loin qu'elles-mêmes le remplaçaient sur place. La traditionnelle répartition femmeintérieur / homme-extérieur n'était pas bouleversée.

8 Le thème de la guerrière connaissait une certaine vogue dans la littérature ou la peinture. Fort apprécié, le mythe - revu et corrigé - des Amazones de l'Antiquité fut utilisé pour glorifier les héroïnes des guerres civiles, présentées par leurs admirateurs comme leurs descendantes chrétiennes, fortes et vertueuses, envoyées par Dieu pour sauver le pays7. Reste que les exemples de nobles dames guerrières renvoient à l'exceptionnel, tant par leur petit nombre que par le contexte particulier des guerres civiles. Idéologiquement aussi, elles se situaient elles-mêmes dans ce registre en assurant qu'elles étaient au-dessus du commun. Sylvie Steinberg a bien analysé ce discours ${ }^{8}$ : c'est leur sang noble qui leur permettrait de dépasser la faiblesse de leur sexe et leur donnerait les mêmes qualités viriles qu'aux Amazones de la fable. Ainsi $\mathrm{M}^{\text {lle }}$ de Montpensier jugeait-elle que ce qui serait défaut chez une autre (amour de la guerre, bravoure, ambition) ne l'était point chez elle grâce à la grandeur de sa naissance. C'est bien ce statut d'exception, personnel et social, qui les rend acceptables dans la société 
d'Ancien Régime fondée sur la principe de l'inégalité, de la distinction: ce qui est valable pour elles ne saurait l'être pour d'autres. Et disparaitra avec la fin des troubles civils.

9 Si la guerre était une affaire d'hommes, l'armée n'était cependant pas un lieu entièrement masculin. Non «professionnalisée» au sens où on l'entendrait aujourd'hui, elle n'était pas coupée de la population et, jusqu'à la fin du XVII ${ }^{e}$ siècle, logeait chez l'habitant; la discipline y était tout à la fois très sévère et relâchée. Et les troupes étaient accompagnées par de nombreux civils, parmi lesquels dominaient les femmes : cantinières et surtout, malgré les multiples interdictions royales ${ }^{9}$, prostituées, épouses ou maîtresses de soldats. Lorsque Watteau, au milieu d'une douzaine de tableaux à thème militaire, représente un bivouac (Le Camp volant, 1709), il peint des soldats au repos, allongés par terre, fumant la pipe, et, au centre de la scène, deux femmes entourées de jeunes enfants, assises près du feu, l'une donnant le sein à un nourrisson ${ }^{10}$. De plus, les historiens ont relevé, dans les archives militaires ou judiciaires, la présence de quelques femmes soldats dans les troupes ${ }^{11}$. Sylvie Steinberg en a ainsi retrouvé quarante-quatre. Toutes se sont engagées, généralement assez jeunes, en se faisant passer pour un homme. Quelques-unes, filles de soldats ou de vivandières, suivent le modèle parental ; d'autres sont orphelines ou en conflit familial. La majorité est originaire de provinces périphériques touchées par le passage des troupes. La plupart disent avoir été poussées par la misère, comme Genevière Grondar (23 ans), engagée en 1710 "par un chagrin qu'elle eut de ce que son mari, elle et ses enfants, ne pouvant plus subsister audit Meudon à cause de la cherté du pain, son mari fut contraint de s'engager dans les troupes. Et quelque temps après, elle s'engagea par désespoir (...) en qualité de dragon, étant vêtue en habit d'homme, ayant jeté ses habits de femme dans la Seine, ce qui fait qu'on croit qu'elle s'est noyée ${ }^{12}$. » Certaines font état de plusieurs mois, voire de plusieurs années, de service avant que leur véritable identité ne soit révélée, souvent à la suite d'une maladie ou d'une blessure. Les autorités militaires se montrent alors assez indulgentes avec elles, du moment que leurs officiers témoignent qu'elles ont toujours eu une " conduite sage ", autrement dit qu'elles se sont conduites comme des soldats et non des prostituées: elles sont renvoyées chez elles et peuvent même parfois obtenir une petite gratification.

En transformant les sujets du roi en citoyens porteurs de droits, en liant port des armes et citoyenneté, la Révolution française remodèle ce paysage.

\section{Port des armes et citoyenneté}

11 Pendant la Révolution, la force publique comprenait deux branches: l'armée, qui pendant la période évolua progressivement en armée de conscription; la garde nationale, formée spontanément en juillet 1789 à partir de l'héritage de la milice bourgeoise ${ }^{13}$ et très vite institutionnalisée. Avec l'entrée en guerre, l'engagement des volontaires et les levées d'hommes, l'armée était certes perçue comme formée de soldats-citoyens. Mais jusqu'en 1795 il n'y avait pas de lien institutionnel entre armée et citoyenneté. D'une part, un citoyen passif ou un étranger pouvaient s'y enrôler. D'autre part, le service militaire ne donnait pas accès au titre de citoyen. Même la Constitution de 1793, qui ouvrait l'exercice des droits de citoyen français à tout étranger domicilié en France depuis un an et y vivant du produit de son travail, ou y étant propriétaire, ou marié à une Française, ou ayant adopté un enfant, ou nourrissant 
un vieillard, ne faisait pas intervenir le service dans l'armée. L'importance prise par la guerre modifia cela: la Constitution de l'an III (1795), qui réintroduit le suffrage censitaire, précise cependant que «sont citoyens, sans aucune condition de contribution, les Français qui auront fait une ou plusieurs campagnes pour l'établissement de la République " (art. 9) ; à l'inverse, " aucun étranger qui n'a point acquis les droits de citoyen français, ne peut être admis dans les armées françaises, à moins qu'il n'ait fait une ou plusieurs campagnes pour l'établissement de la République » (art. 287).

En revanche, la garde nationale était explicitement liée au droit de cité : servir dans la garde nationale et voter dans les assemblées primaires étaient les droits politiques du citoyen, définissant la citoyenneté. Ainsi, dans les années de suffrage censitaire (avant 1792 et après 1795), seuls les citoyens actifs avaient le droit (et le devoir, car c'était une obligation) de porter les armes dans la garde nationale. «ÊTRE ARMÉ POUR SA DÉFENSE PERSONNELLE EST LE DROIT DE TOUT HOMME INDISTINCTEMENT ; ÊTRE ARMÉ POUR LA DÉFENSE DE LA PATRIE EST LE DROIT DE TOUT CITOYEN », AFFIRMAIT ROBESPIERRE EN 1791. EN 1792, L'ABOLITION DU SUFFRAGE CENSITAIRE FUT PRÉCÉDÉE PAR L'ENTRÉE DE FAIT DES «PASSIFS» DANS LA GARDE NATIONALE. LE DÉSARMEMENT DE SANS-CULOTTES EN 1795 FUT VÉCU PAR EUX COMME UNE «FLÉTRISSURE POLITIQUE», LOGIQUEMENT ACCOMPAGNÉE PAR L'INTERDICTION D'ASSISTER AUX ASSEMBLÉES SECTIONNAIRES.

13 Exclues du droit de cité, les femmes ne pouvaient ni voter dans les assemblées ni servir dans la garde nationale. Elles furent très peu nombreuses à réclamer publiquement le droit de suffrage ${ }^{14}$. En conclure que seule une petite poignée d'entre elles auraient demandé l'accès à la citoyenneté tandis que les autres auraient été complètement insensibles à la question, c'est plaquer sur la Révolution une conception de la citoyenneté - réduite au droit de vote - qui est la nôtre. Et c'est donc ne pas tenir compte des demandes de femmes à être organisées en garde nationale. Présentes dès 1791, elles s'amplifièrent au moment de l'entrée en guerre (20 avril 1792). En ce sens, elles participaient indéniablement au grand élan patriotique de 1792. Mais celui-ci était inséparable d'une redéfinition active de la citoyenneté, portée par le mouvement populaire et qui conduisit à l'abolition entre "passifs» et «actifs $»^{15}$. Découlant du désir de défendre la nation en révolution, les demandes féminines d'armement le dépassaient pour s'inscrire dans la problématique des droits politiques. Si elles ne revendiquaient pas un statut plein et entier de citoyennes, ces femmes souhaitaient au moins une entrée implicite dans la citoyenneté.

14 Dans plusieurs villes de province, certaines prononcèrent des discours en ce sens et formèrent parfois d'éphémères «compagnies d'amazones", "de citoyennes françaises ${ }^{16}$. À Paris, le 6 mars 1792, une militante populaire, Pauline Léon, présentait à la Législative une pétition signée par plus de 320 Parisiennes qui sollicitaient la permission d'organiser une garde nationale féminine ${ }^{17}$ : se revendiquant citoyennes, elles réclamaient leur armement au nom du droit naturel "qu'a tout individu de pourvoir à la défense de sa vie et de sa liberté », au nom de la Déclaration des Droits dont on ne pouvait prétendre, disaient-elles, qu'elle « n'a point d'application pour les femmes ». Vingt jours plus tard, dans une société populaire, Théroigne de Méricourt invitait les citoyennes (le mot ponctue son discours) à s'organiser en corps armé ; elle développait point par point les mêmes thèmes et concluait par un appel qui dit clairement le lien entre port des armes et aspiration à l'égalité politique : «Brisons nos 
fers, il est temps enfin que les femmes sortent de leur honteuse nullité où l'ignorance, l'orgueil et l'injustice des hommes les tiennent asservies depuis si longtemps ${ }^{18}$.» En juillet de nouveau, quatre-vingts pétitionnaires demandaient aux députés de décréter l'armement des « vraies citoyennes » pour défendre la capitale, arguant de ce que, elles, femmes libres, n'avaient pas moins de courage que Jeanne d'Arc qui avait sauvé la France sous le règne d'un despote ${ }^{19}$.

Les réactions masculines à ces demandes sont assez éloquentes, conjuguant éloges de rigueur devant leur patriotisme et ferme rappel du fait que le port des armes est réservé aux hommes et doit le rester. Si le président de la Législative loua l'enthousiasme des pétitionnaires du 6 mars - dont le principal intérêt aurait été de ranimer les vertus civiques des « hommes faibles »-, le député Dehaussy-Robecourt les mit en garde : «Gardons-nous d'intervertir l'ordre de la nature ; elle n'a point destiné les femmes à donner la mort ; leurs mains délicates ne furent point faites pour manier le fer, ni pour agiter des piques homicides. » L'Assemblée passa alors à l'ordre du jour. Cinq mois plus tard, Pauline Léon subit une autre désillusion : alors que, lors de l'insurrection du 10 août 1792, elle avait rejoint le bataillon de sa section une pique à la main, elle dut, "à la prière de presque tous ces patriotes ", la céder à un sans-culotte ${ }^{20}$. Cela ne découragea pas la jeune femme, que l'on retrouve - avec plusieurs des pétitionnaires du 6 mars 1792 - parmi les fondatrices du club des Citoyennes Républicaines Révolutionnaires. Politiquement très radical, celui-ci fut créé en mai 1793 par des Parisiennes avec, à l'origine, l'objectif de former des "compagnies d'amazones ", composées de toutes les femmes de 18 à 50 ans, chargées de "garder l'intérieur, tandis que leurs frères garderont les frontières ». Ainsi transposaient-elles la traditionnelle coupure homme-extérieur / femme-intérieur sur le terrain politique de la défense armée de la Révolution! Il va sans dire que cela fut assez mal accepté. Lorsque la jeune Société se présenta aux Jacobins le 10 mai 1793, elle fut d'abord applaudie, puis les choses se gâtèrent lorsque l'oratrice exposa leur programme : «bruit... bruit... », liton sur le procès-verbal de la séance ${ }^{21}$. Le terme est pudique pour désigner ce qui fut manifestement une franche hostilité car c'est en sanglotant que l'oratrice termina son discours : «Nous sauverons la patrie, citoyens, ne croyez pas nous décourager. " Les Citoyennes Républicaines Révolutionnaires jouèrent un rôle très important dans le mouvement révolutionnaire de l'été $1793^{22}$; mais, bien que le règlement de la Société, daté du 9 juillet 1793, signala encore que son but était « de s'armer pour concourir à la défense de la Patrie », il ne fut plus question dans leurs interventions d'armer les femmes.

16 Le problème fut cependant de nouveau soulevé à l'automne 1793. À la suite d'une campagne des militantes révolutionnaires, la Convention avait voté le 21 septembre 1793 une loi obligeant les femmes à porter, comme les hommes, la cocarde tricolore, symbole de citoyenneté. Alors que les revendications féminines se faisaient plus insistantes, la mesure provoqua les rumeurs les plus folles ${ }^{23}:$ on disait dans les rues de Paris que, poursuivant sur leur lancée, les femmes allaient se couper les cheveux, porter le bonnet rouge, demander et obtenir les armes - ce qui serait "humiliant " pour le sexe masculin -, puis le droit de suffrage. On le voit : c'était bien des droits politiques dont il s'agissait, et de la définition du masculin et du féminin (cheveux courts). Le 8 brumaire an II (29 octobre 1793), le député Fabre d'Églantine brandissait à la Convention le spectre d'un armement féminin. Après un violent réquisitoire contre les Citoyennes Républicaines Révolutionnaires, décrites comme «une sorte de chevaliers errants, (...) des filles émancipées, des grenadiers femelles ${ }^{24}$, il revenait sur 
les angoisses exprimées depuis fin septembre : elles avaient obtenu la cocarde, bientôt elles allaient " demander la ceinture, puis les deux pistolets à la ceinture ». Il prévenait ses collègues: "Vous verriez des files de femmes allant au pain comme on va à la tranchée. » Pis : les «malveillants » sauraient fort bien utiliser contre la Révolution les armes dont elles seraient, elles, incapables de se servir. Le même jour, dans un cabaret, des clients - peut-être un peu éméchés - s'inquiétaient de ce que, une fois armées, les femmes pourraient assassiner les hommes « au moment où ils n'y penseraient pas, (... puis...) laisseraient régner (...) une Catherine de Médicis qui enchaînerait les hommes $\aleph^{25}$. Juxtaposés au discours de Fabre d'Églantine, ces propos soulignent la hantise d'un armement féminin, vécu comme un pas vers l'« émancipation ", l'égalité politique et la confusion des $\operatorname{sexes}^{26}$, et conduisant à la ruine de la société et de la Révolution, voire à la destruction des hommes.

Le lendemain du discours de Fabre d'Églantine, les clubs de femmes étaient interdits et le député Amar réaffirmait avec force qu'elles ne pouvaient exercer les droits politiques. Le vent avait tourné et, que ce soit pour le revendiquer ou pour le craindre, on ne parla plus désormais d'un armement politique des citoyennes à l'intérieur du pays.

18 À côté de ces demandes - qui furent de toute façon minoritaires - et des réactions qu'elles ont suscitées, s'est aussi posée la question du service des femmes à l'armée. Ne renvoyant pas de la même façon aux droits du citoyen, les termes en furent beaucoup moins violents, même si le sujet touchait aussi à la conception de la différence des sexes.

\section{La « gloire » de se battre pour la République}

Illustrateur des aspects populaires de la Révolution, l'aquarelliste Béricourt a peint un Camp républicain ${ }^{27}$ qui met en scène hommes, femmes et enfants. Devant un arrière-plan formé par les tentes figurent les trois groupes : cinq très jeunes enfants jouant autour d'un feu; cinq femmes entourant une autre en train d'accoucher sous un arbre tandis qu'une septième berce son propre nourrisson; cinq soldats en train de trinquer, semble-t-il pour fêter l'heureux événement. La représentation d'un accouchement souligne bien que la vie dans les armées républicaines mêlait civils et militaires, hommes et femmes, masculin et féminin. Les autorités visitant l'armée le notaient aussi : «la quantité de femmes est effrayante », « les cantonnements sont engorgés de femmes ", etc ${ }^{28}$. Comme autrefois dans les armées du Roi, on trouve parmi ces femmes des prostituées, quelques réfugiées de province envahies, des vivandières et surtout des maitresses ou épouses d'officiers et de soldats. Celles-ci étaient peut-être plus nombreuses que pendant l'Ancien Régime car, à l'instar du tambour-maître Perrinet parti avec sa femme et ses trois enfants « qui n'avaient alors aucune ressource dans leur domicile $»^{29}$, des sans-culottes engagés volontaires trouvaient normal d'emmener avec eux leur famille - surtout avant que ne fut prise la décision de verser des secours aux familles des soldats. Des femmes décidaient aussi de rejoindre leur mari parti depuis plusieurs mois, parce qu'elles étaient sans ressources, désiraient le revoir ou le secourir : en février 1793, Elisabeth Françoise Dubois (30 ans), épouse du capitaine des canonniers Favre, "ayant appris que son mari, ainsi que les autres défenseurs des frontières manquaient de tout en vêtement, linge et chaussures, (...) se détermina à faire les plus grands sacrifices pour porter des secours à son mari ; qu'en conséquence 
elle vendit la majeure partie de ses effets pour se rendre auprès de lui » à Lièges et lui porter "un nouveau trousseau " et du numéraire ${ }^{30}$. Plusieurs de ces femmes liaient amour de leur mari et amour de la Patrie: elles ne souhaitaient pas seulement accompagner un époux soldat, elles voulaient être avec celui et ceux se battant pour la Révolution, participer à leurs victoires et à leurs revers. La citoyenne Rousseau, ouvrière, partit en Flandre avec son ami engagé volontaire : «je suivrai mon amant, puisqu'il combat pour la patrie. (...) nous reviendrons triomphants ou nous mourrons tous les deux dans les combats. ${ }^{31}$ " À l'armée, elle gagna sa vie en faisant la cuisine et en réparant les uniformes; d'autres furent blanchisseuses (citoyenne Perrinet) ou cantinières. Quant à la femme Favre, une fois arrivée à Lièges, « l'amour de sa patrie lui mit les armes à la main » : nommée capitaine en second par la compagnie de son mari, elle fit le siège de Maëstricht, bivouaqua dans la neige et fut faite prisonnière le $1^{\mathrm{er}}$ mars 1793.

Comme elle, plusieurs femmes combattirent, sous des uniformes aussi variés que ceux de canonniers, grenadiers, gendarmes, fusiliers, chasseurs... Les historiens $\mathrm{s}^{32}$ en ont recensé environ 80 dans les archives parlementaires, militaires, policières. Ce chiffre est bien entendu un minimum: sans être des milliers, elles furent cependant plus nombreuses que dans les armées royales. Il n'est pas rare que des témoins évoquent leur présence ; et, surtout, il semble que leur existence frappe car on parle d'elles, on les représente sur des gravures ou des caricatures ${ }^{33}$, on les met en chansons. Fin 1792début 1793, paraît une brochure intitulée Départ de Neuf Cent (sic) Citoyennes de Paris, Qui se sont enrôlées, déguisées en hommes, pour partir aux Frontières combattre les Tyrans des Nations, signée Manette Dupont ${ }^{34}$. Elle demande aux Conventionnels de créer un "nouveau corps de défenseurs de la République française de citoyennes": ce "corps de Fernigh $»^{35}$ (sic) serait formé de 10000 volontaires âgées de 18 à 40 ans, réparties en 5 légions (20 bataillons et 100 compagnies). Chaque légion aurait un uniforme différent, minutieusement décrit dans la pétition, et serait dotée d'une devise inscrite sur le drapeau tricolore (Tout est égal chez nous pour vaincre les tyrans; Femmes libres; Vivre libre ou mourir; La République, ou la mort; Ça ira). Toutes auraient les cheveux courts afin de ne pas perdre « de tems à une toilette qui ne sert qu'à fasciner les yeux, et qui devient fort inutile à des citoyennes, dont le but est d'être invincibles». Il est difficile de mesurer l'authenticité de ce texte: si ce ne sont certainement pas 900 citoyennes enrôlées qui en sont à l'origine, il ne ressemble pas non plus aux pastiches tournant en dérision les revendications féminines ${ }^{36}$. Prônant également des mesures que n'auraient pas reniées les sans-culottes (imposition des riches...), il s'adresse aux femmes du peuple et aux « femmes libres ». L'ensemble laisse penser qu'il s'agit bien d'un écrit de femme, reflet radicalisé de certaines aspirations féminines, et qui par deux fois rappelle, comme un fait très connu, que des citoyennes se sont déjà enrôlées dans les armées.

On possède quelques éléments biographiques, assez disparates et lacunaires, sur quarante-quatre d'entre elles. Frappe d'abord leur jeunesse : la moitié des dix-huit dont on connaît l'âge ont moins de 20 ans lors de leur engagement (à peine 15 ans pour quatre d'entre elles), six ont entre 20 et 22 ans et trois entre 30 et 48 ans; deux se disent « enfants de troupe » et ont probablement grandi dans les camps. Huit précisent s'être enrôlées sous une identité masculine, mais la majorité ne cachent pas leur sexe. $\mathrm{Au}$ moins seize ont un ou plusieurs parents servant dans le même bataillon, que ce soient un mari (11), des frères ou un père (8), la plupart enrôlés volontaires en 1792 
pour défendre la Patrie en danger : ce sont là des familles animées par l'esprit révolutionnaire plus que par un modèle militaire. Avant de faire la guerre, Marie Charpentier avait d'ailleurs pris part au siège de la Bastille, Catherine Pochetat (22 ans) à ceux de la Bastille et des Tuileries, Josèphe Heurtet femme Communeau fréquentait le club des Cordeliers ${ }^{37}$.

Chez celles qui partent se mêlent goût de l'aventure, sentiments patriotiques et désir de ne pas laisser aux hommes la gloire de se battre pour la Révolution. Mariée au volontaire Henry, Rose Bouillon, « applaudissant au patriotisme de son mari, et voulant de même contribuer à l'affermissement de la République, laissa deux enfants dont l'un âgé de sept mois aux soins de sa mère, changea ses habits de femme en habits d'homme et vint rejoindre son mari $»^{38}$; inscrite en mars 1793 comme volontaire au bataillon, elle combattit jusqu'à la mort d'Henry en août 1793, puis rentra chez elle s'occuper de ses enfants. «Enflammée du feu sacré de la liberté, encouragée par l'exemple précieux de cinq frères » volontaires, Reine Chapuy (17 ans) s'engagea en février 1793 dans un régiment de cavalerie: «j'aurais cru déroger au sang généreux qui coule dans mes veines et dans celles de toute ma famille si je n'avais fait le sacrifice des alarmes, qui sont le partage ordinaire de mon sexe, au désir brûlant de combattre les tyrans et de partager la gloire de les foudroyer. » Revenue en décembre, elle demandait quelques semaines plus tard l'autorisation de repartir : « serait-ce à la fleur de mes ans que je me verrais réduite à aller habiter les foyers paternels, tandis que Bellone m'attend dans les siens (...) ? Ah! mes frères, vous qui avez le bonheur de combattre, lorsque vous reviendrez couverts de gloire comment accueillerez-vous votre sœur infortunée? De quel œil me regarderez-vous? C'est donc en vain que j'avais, à votre exemple, fait le serment de mourir pour la République ? (...) Que ma demande me soit accordée (...) et je prouverai que le bras d'une femme vaut bien celui d'un homme, lorsque ses coups sont dirigés par l'honneur, la soif de gloire, et la certitude d'exterminer les grands. ${ }^{39}$

Sur place, elles étaient en général assez bien considérées par leurs compagnons d'armes, dont elles partageaient le sort, participant aux sièges et aux combats : à SaintPierre-Chemillé, la femme Communeau fit mordre la poussière à trois "brigands » vendéens ; à Hondschoote, la jeune Anne Quatresous (15 ans) eut deux chevaux tués sous elle, etc ${ }^{40}$. Leurs maris tombés à leurs côtés, Rose Bouillon, Rose Liberté Barreau et la femme Soutemanne restèrent à leur poste et continuèrent à faire feu. Six affirment avoir été à Jemmapes et quatre à Maëstricht; au moins dix ont été sérieusement blessées et trois faites prisonnières ${ }^{41}$. Revenant des frontières à la fin de l'an II, la citoyenne Degressain (30 ans) était, selon des témoins, «mutilée de cicatrices » : elle en avait une à la lèvre supérieure, une au coin de l'œil, plusieurs sur les cuisses et n'avait plus qu'une seule oreille ${ }^{42}$. Plusieurs reçurent des attestations de bravoure de leurs chefs. Certaines devinrent officiers ou sous-officiers, élues par leurs camarades ou nommées par leurs supérieurs : on relève ainsi un capitaine en second (femme Favre), un lieutenant (Ursule Aby), trois sous-lieutenants (Catherine Pochetat, Pélagie Dulière, Marie Schellinck), deux sergents (Marie Charpentier et Angélique Duchemin). Ces promotions ne plaisaient pas à tous : en septembre 1793, un citoyen s'indignait ainsi de l'existence d'une femme officier à l'Armée du Nord, assurant que « les soldats ont tous les jours la honte de recevoir ses ordres, ce qui leur déplaît infiniment $»^{43}$; et il dénonçait cette situation comme illégale.

En effet, le 30 avril 1793, la Convention avait voté un décret arrêtant que «toutes les femmes inutiles au service des armées » devaient quitter les camps et cantonnements 
sous les huit jours. Il faisait suite aux rapports sur le nombre impressionnant de femmes qui suivaient les bataillons, absorbaient une partie des subsistances, gênaient la marche des troupes et le cours des opérations. Leur était aussi reproché de «semer la terreur dans les camps », de divertir les soldats de leur devoir, de les « amollir », de les rendre " efféminés ", lâches, sans vigueur ${ }^{44}$. Ces remarques visaient le fléau bien réel de la syphilis ${ }^{45}$. Mais le vocabulaire utilisé fait irrésistiblement penser aux discours, qui s'étaient imposés dans la seconde moitié du XVIII ${ }^{e}$ siècle, sur la nécessaire séparation des sexes ${ }^{46}$. Leurs auteurs dénonçaient la place des femmes dans les cercles de pouvoir, à la Cour, dans les salons, et assuraient que l'intrusion du féminin dans l'espace public dévirilisait les hommes, les rendant efféminés, sans vigueur (le roi), sans génie (les écrivains) : ici, pour les soldats, sans vaillance et courage.

Le décret du 30 avril 1793 prévoyait que seules pourraient demeurer à l'armée les vivandières et les blanchisseuses, dont le nombre était limité. Son avant-dernier article (XI) précisait que « les femmes qui servent actuellement dans les armées seront exclues du service militaire; il leur sera donné un passeport et 5 sous par lieue pour rejoindre leur domicile ». Ce point n'avait pas été abordé dans le rapport précédant le vote : sans aucun considérant explicatif, il était comme glissé dans ce décret visant les «femmes inutiles au service ». Or, à la différence des épouses et des prostituées, les femmes soldats étaient peu nombreuses; d'un simple point de vue matériel, elles ne gênaient pas l'armée, bien au contraire. Leur exclusion était donc de l'ordre du symbolique. Elle n'avait pas pour but de faire de l'armée un lieu entièrement masculin, puisque restaient les blanchisseuses et les vivandières - dont les tâches auraient concrètement très bien pu être remplies par des hommes. En ce temps de révolution, de ruptures et de possibles, elle était prise pour rappeler que la radicale réorganisation de la société ne devait pas s'étendre à la différence masculin-féminin. Le port des armes appartient au masculin : les soldats doivent être des hommes. Le rôle nourricier et le soin des corps relèvent du féminin : les vivandières et blanchisseuses sont des femmes.

Mais l'écart est souvent grand entre les théories, les normes reposant sur les représentations, et les pratiques : le décret du 30 avril 1793 ne fut guère appliqué. Les compagnes de soldats continuèrent à suivre les troupes, les généraux n'étant d'ailleurs pas les derniers à contrevenir à la loi. Et la majorité des combattantes continuèrent à se battre. Je n'en ai trouvé que trois quittant l'armée en mai ou juin 1793 ; et seule l'une d'entre elles dit explicitement avoir été renvoyée suite au décret. Certes, plusieurs furent congédiées à cause de leur sexe, mais des mois, voire des années, après le 30 avril $1793^{47}$ - certaines, il est vrai, étant cachées sous une identité masculine. Faisant état de leurs anciens faits d'armes, les citoyennes Chapuy et Pochetat demandèrent à la Convention la permission de retourner se battre: elles furent applaudies, on leur accorda une gratification, et on passa à l'ordre du jour sur leurs pétitions ${ }^{48}$. La plupart poursuivirent cependant leur service comme si de rien n'était; quelques-unes s'engagèrent même après avril 1793. Par méconnaissance ou mauvaise volonté, leurs chefs ignoraient tout bonnement la loi : le 8 juin 1793, le capitaine de la femme Fortier l'autorisait par écrit à " être conservée » dans sa compagnie de canonniers ${ }^{49}$. Fille d'un maître d'école, Félicité Duquet ( 21 ans) s'était travestie en homme et enrôlée le 7 septembre 1792, sous le surnom de Vadeboncœur; les fatigues de la guerre la poussèrent à révéler son identité pour demander un congé, qui lui fut accordé en février 1793. En juillet 1793, souhaitant reprendre du service, elle fut incorporée par le général Ferrand dans le $1^{\text {er }}$ bataillon de la Nièvre, avec lequel elle combattit comme fusilier jusqu'à ce que, le 18 avril 1794 - un an après le décret! -, le conseil 
d'administration du bataillon s'interroge sur la légalité de sa présence : un membre ayant observé qu'elle devrait être renvoyée, le conseil, « considérant que la citoyenne Vadeboncœur s'est toujours comportée (...) d'une manière irréprochable et qu'il n'a aucune connaissance de la loi qui la met dans le cas d'être exclue du bataillon ", va demander son avis au général. Celui-ci, "ayant parfaite connaissance de la loi sur l'exclusion des femmes", ordonne qu'elle soit "renvoyée sur le champ»: elle est congédiée le 5 mai $1794^{50}$.

Certaines étaient encore là sous le Directoire ou l'Empire, comme la célèbre Thérèse Figueur, dite Sangène, qui combattit avec ses frères de 1792 à 1801, ou Angélique Duchemin veuve Brulon : «fille, sœur et femme de soldat, née [en 1771] au milieu des camps ", cette dernière fut autorisée à servir après la mort de son mari en 1792, et ce jusqu'en 1799, quand, grièvement blessée, elle fut admise aux Invalides; elle fut nommée sergent pendant ses campagnes, puis sous-lieutenant en 1822 et, à la fin de sa vie, reçut de Louis-Napoléon Bonaparte la croix de la Légion d'honneur et la médaille de Sainte-Hélène ${ }^{51}$.

D'autres se reconvertirent en vivandières. Ainsi Elisabeth Bourgès, enrôlée en novembre 1792 sous le pseudonyme de Nicolas Sans-Soucy: après que sa véritable identité eut été découverte, elle quitta l'armée le 7 avril 1793, mais y retourna peu après et obtint deux gratifications en 1794 et 1795 pour avoir sauvé des blessés, qu'elle avait pansés avec sa propre chemise ou portés sur son dos jusqu'aux voitures d'ambulance ${ }^{52}$. Anciennes soldates ou non, les cantinières étaient présentes sur les champs de bataille, distribuant de l'eau-de-vie, soignant les blessés, n'hésitant pas à faire feu au besoin. Les archives militaires attestent de la conduite héroïque de plusieurs d'entre elles et la légende raconte que celle appelée Marie-Tête-de-Bois - née et élevée aux Invalides - fit dix-sept campagnes au cours desquelles elle se battit plusieurs fois, accoucha en pleine bataille de Marengo et aurait été dans le dernier carré des braves à Waterloo ${ }^{53}$.

Plusieurs aussi quittèrent l'armée, non pas à cause du décret du 30 avril 1793, mais volontairement, parce que, après une ou plusieurs campagnes ${ }^{54}$, elles étaient mutilées, blessées, fatiguées. Bien qu'ayant contrevenu à la loi en ayant continué à servir, elles étaient reçues avec les honneurs dans les assemblées révolutionnaires, et obtenaient généralement une gratification ou, mieux mais plus rare, une pension comme ancien soldat.

30 L'attitude des autorités envers elles était ambivalente, balançant entre admiration et volonté de maintenir la distinction entre qualités masculines et qualités féminines. Or, la force, le courage, l'énergie, l'audace seraient propres aux hommes, la Nature ayant en revanche doté les femmes de plus de douceur et de dévouement privé. Cette conception, qui ne date pas de la Révolution, est longuement développée dans le rapport d'Amar pour justifier l'interdiction des clubs de femmes et leur exclusion du droit de cité. Or, l'existence et la conduite des femmes soldats dérangeaient cette représentation de la différence des sexes. Aussi furent-elles souvent présentées comme des miracles de la Liberté et de la haine des tyrans, capables d'élever des femmes « audessus » de leur sexe, de les doter de qualités "masculines", de les transformer en hommes: «Je ne la range même pas parmi les femmes, mais je déclare que cette fille est un mâle, puisqu'elle a, comme les plus intrépides guerriers, affronté la mort dans toutes les circonstances périlleuses ", proclamait le dirigeant Collot d'Herbois le 26 février 1794 à propos de la jeune Anne Quatresous, aux faits d'armes particulièrement 
impressionnants ${ }^{55}$. L'héroïsme guerrier ne pouvait être que masculin, même s'il était illustré par une femme.

31 Pour le rédacteur des Révolutions de Paris, contempteur toujours virulent de toute intervention des citoyennes dans la sphère publique, l'héroïsme au féminin ne devrait être que celui du quotidien, modeste et domestique: en janvier 1793, après avoir soutenu que «les femmes n'ont rien à faire à l'armée » car, partout ailleurs que dans leurs foyers, elles sont "déplacées", il s'adresse à elles $^{56}$ : «Laissez-nous le fer \& les combats ; vos doigts délicats sont faits pour tenir l'aiguille \& semer de fleurs le chemin épineux de la vie. Pour vous, l'héroïsme consiste à porter le poids du ménage \& les peines domestiques. »

La position des dirigeants révolutionnaires était plus ouverte : ils saluaient la bravoure des femmes soldats tout en insistant sur les rôles féminins. Ainsi le Recueil des Actions héroïques et civiques, publié en l'an II par le Comité d'Instruction publique de la Convention pour proposer des modèles aux jeunes Français, ne les oubliait-il pas : à côté de la citoyenne dévouée, qui a "sacrifié " sa jeunesse pour soigner une mère atteinte d'une « maladie rebutante », figurent deux combattantes, Rose Liberté Barreau et Rose Bouillon ${ }^{57}$. Toutes deux ont en commun d'avoir servi avec leurs maris et, surtout, d'avoir poursuivi le combat après qu'ils aient été touchés à côté d'elles. Le Recueil glorifie cela, en ajoutant que ni l'une ni l'autre n'a « renoncé aux vertus de son sexe »: Liberté Barreau soigne son époux après la victoire et Rose Bouillon retourne prendre soin de ses enfants après la mort du sien. Et si le récit célèbre clairement l'héroïsme militaire de Liberté Barreau, la gravure qui l'accompagne apporte une touche d'ambiguïté : elle n'y est pas représentée en train de combattre, de recharger son fusil avec les cartouches de l'Espagnol qu'elle vient d'abattre ou de poursuivre l'ennemi en déroute, mais portant secours à son mari qu'elle entoure de ses bras "Actuellement qu'ils mordent la poussière, je te dois tous mes soins ", est-il écrit sous la gravure.

Ajoutons, avant de conclure, que l'Armée catholique et royale présentait, de ce point de vue, des similitudes avec les armées républicaines. Elle était suivie par des cohortes de femmes, d'enfants et de vieillards fuyant les régions dévastées par les Bleus. Elle comptait également quelques combattantes, dont la plus célèbre est Renée Bordereau, dite Brave l'Angevin: selon ses Mémoires (1814), cette paysanne, héroïne de la geste vendéenne, aurait fait toutes les guerres de Vendée et tué un moins une vingtaine de Bleus. La marquise de la Rochejaquelein écrit qu'elles auraient été une dizaine, sans compter les quelques nobles se battant avec leur mari - telle $\mathrm{M}^{\mathrm{me}} \mathrm{du}$ Bruc, tuée en combattant en février 1794 près de Beaupréau. Toujours d'après la marquise, la présence de femmes, soldats ou non, aurait été combattue par les généraux qui " avaient plusieurs fois déclaré qu'ils feraient tondre et chasser la première femme, déguisée ou non, qui suivrait l'armée $\aleph^{58}$.

\section{Conclusion}

Sous la monarchie, le port de l'épée était un des privilèges nobiliaires. Avec la Révolution, le port des armes dans la garde nationale devint un des droits politiques du citoyen. Aussi, les demandes d'armer les femmes à l'intérieur du pays, si elles procédaient assurément d'un sentiment patriotique, ont-elles été perçues, par celles qui les formulaient et par ceux qui les rejetaient, comme une voie d'accès à la 
citoyenneté. Il est indispensable de ne pas l'oublier pour saisir pleinement la portée de ces revendications. Mais il importe également de ne pas confondre garde nationale et armée, hypothétique organisation de citoyennes armées et enrôlement militaire de femmes soldats. Certes, la coupure entre les deux n'était pas absolue et les refus opposés à l'une comme à l'autre reposaient sur d'identiques conceptions du masculin et du féminin. Même avant qu'il ne soit formalisé par la Constitution de l'an III, le lien entre service aux armées et citoyenneté existait indéniablement : épaulée par un de ses camarades de bataillon, la femme Favre s'étonnait ainsi en 1793 qu'on lui ait refusé une carte de citoyen ${ }^{59}$. Reste que l'on n'est pas tout à fait dans le même espace. Les pétitionnaires de 1792 ou les Citoyennes Républicaines Révolutionnaires demandaient, collectivement, l'armement des civiles comme un droit du citoyen, garanti par la Déclaration des Droits. L'enrôlement de soldates était quant à lui un acte individuel ; leurs auteurs ne revendiquaient pas l'ouverture de l'armée à l'ensemble des femmes. Quand elles expliquent leur geste, elles ne font pas référence à leur titre de citoyennes qui leur donnerait des droits, mais à leur amour de la Patrie qui les pousse à dépasser la faiblesse de leur sexe. Dans un cas il est question de l'accès des - et non pas d'une femmes à la citoyenneté, pas dans l'autre.

Plus généralement, l'identification de l'armée au masculin se fait à plusieurs niveaux. Est parfois dit que les femmes ne sont pas faites pour donner la mort. Dans leurs peintures d'un camp de soldats, Watteau et Béricourt les représentent tous deux donnant la vie, par l'allaitement ou l'accouchement. Plus fréquemment, est mise en avant leur constitution fragile et délicate, à laquelle est associée la douceur. Ces " caractéristiques » sont pensées comme inverses - et complémentaires - des qualités définissant l'homme, et le soldat : force et courage. Renvoyant également à une série de schèmes de pensée stéréotypés, est souvent sous-jacente l'idée que, si à la rigueur une femme peut combattre pour sa défense et celle des siens, la conquête relève du masculin $^{60}$. La conquête militaire, c'est aussi la gloire du héros: le mot revient constamment, notamment dans les propos de combattantes révolutionnaires, qui disent vouloir partager avec les hommes «la gloire de se battre ». Le couple défense/ conquête renvoie à un autre : intérieur/extérieur. Or le genre féminin est associé à l'intérieur (ce qui, à l'époque moderne, ne se réduit pas au privé): raison supplémentaire pour que le soldat, qui combat hors du pays, ne soit pas une femme. Les guerres civiles brouillent ce schéma et la Révolution le complexifie en faisant du révolutionnaire armé le défenseur politique de l'intérieur du pays : inscrite pourtant dans une symbolique traditionnelle, la proposition des Républicaines Révolutionnaires de faire garder l'intérieur par les femmes et les frontières par les hommes ne pouvait qu'être mal accueillie.

«Je laisse ma quenouille et mes fuseaux à celui qui sera assez lâche pour rester dans ses foyers et je me charge de prendre son fusil et son sabre pour remplir sa place", s'exclamait Marie Morel après s'être enrôlé en juin $1793^{61}$. Malgré des contextes différents, la logique est la même que celle sous-tendant les gravures sur le monde à l'envers, les propos de $\mathrm{M}^{\mathrm{me}}$ de la Guette ou de Collot d'Herbois. Dans tous les cas, guerre, armes et bravoure restent du côté du masculin, le féminin est déprécié et la hiérarchie des genres maintenue. À travers les siècles, les représentations ont la vie dure. Mais elles ne sont pas immuables: "le bras d'une femme vaut bien celui d'un homme ", proclame Reine Chapuy en 1793. En 1805, $\mathrm{M}^{\mathrm{me}}$ de Xaintrailles, qui a fait sept campagnes et participé à l'expédition d'Égypte comme aide de camp, demande 
«justice " à Napoléon alors qu'on lui refuse une retraite "parce qu'elle est femme »: « mais j'étais femme, quand j'ai repris aux Prussiens..., quand j'ai empêché la révolte..., quand j'ai sauvé le $11^{\mathrm{e}}$ bataillon..., quand j'ai pansé les blessés... J'étais femme aussi quand... ». Et elle conclut : « Ce n'est point en femme que j'ai fait la guerre, je l'ai faite en brave. ${ }^{62}$ » On n'est plus là dans un système de hiérarchie, mais d'égalité (une femme vaut un homme), voire d'indifférenciation fonctionnelle (un brave est un brave, quel que soit son sexe). Ces voix discordantes méritaient d'être signalées.

\section{BIBLIOGRAPHIE}

\section{Sources}

Archives parlementaires de 1787 à 1860 : recueil complet des débats législatifs et politiques..., 1867-2004, $1^{\text {ère }}$ série (1787-1799), Paris, P. Dupont, puis CNRS.

Les Femmes dans la Révolution (recueil de textes), 1982, Paris, Edhis, 2 vol.

Guette $\mathrm{M}^{\mathrm{me}}$ de la, 1982 (1 $1^{\text {ère }}$ ed. 1681), Mémoires, (éd. M. Cuénin), Paris, Mercure de France.

Le Moniteur Universel (Réimpression), 1840-1845, Paris.

Recueil des Actions hérö̈ques et civiques des républicains français, présenté à la Convention nationale au nom de son comité d'instruction publique, par Léonard Bourdon, an II, Paris, Imprimerie nationale.

Rousseau Jean-Jacques, 1966 (1ère ed. 1762), Émile ou de l'éducation, Paris, Garnier-Flammarion.

\section{Travaux}

Applewhite Harriet et Lévy Darline, 1990, «Women, Radicalization, and the Fall of the French Monarchy ", in Applewhite Harriet \& Lévy Darline (éd.), Women and Politics in the Age of the Democratic Revolution, Ann Arbor, University of Michigan Press, pp. 81-107.

Bertaud Jean-Paul, 1979, La Révolution armée. Les soldats-citoyens et la Révolution française, Paris, Robert Laffont.

-1983, La Vie quotidienne des soldats de la Révolution française, Paris, Hachette.

Brice Raoul, 1913, La Femme et les Armées de la Révolution et de l'Empire, Paris, L'Édition moderne.

Chassin Charles Louis et Hennet Léon, 1899, Les Volontaires nationaux pendant la Révolution

française, Paris, Quantin, t. III.

Corvisier André, 1964, L'Armée française de la fin du XVII siècle au ministère de Choiseul. Le soldat, Paris, PUF.

Cuénin Michèle, 1992, La Dernière des Amazones. Madame de Saint-Baslemont, Nancy, Presses universitaires de Nancy.

- 1987, « Les femmes et la guerre, 1516-1660 » in Ian Richmond et Constant Venesoen (dir.), Présences féminines, Seattle.

Day-Hickman Barbara, 2004, «Heroines or Harlots : Women Combatants during the Revolutionary Wars ", Communication au Congrès annuel de la Society of French Historical Studies, Paris. 
Dekker Rudolf, 1989, « Republican Heroïnes : Cross-Dressing Women in the French Revolution Armies ", History of European Ideas, vol. $10 \mathrm{n}^{\circ}$ 3, pp. 353-363.

Duby Georges et Perrot Michelle (dir.), 1991, Histoire des femmes en Occident, t. III XVI ${ }^{e}$-XVIII ${ }^{e}$ siècles (dir. Zemon Davis Natalie et Farge Arlette), Rome-Bari, Laterza / Paris, Plon.

Farge Arlette, 1996, Les Fatigues de la guerre, Paris, Gallimard.

Gerbaux François, 1904, « Les femmes soldats pendant la Révolution », La Révolution française, t. 47, pp. 47-61.

Godineau Dominique, 1988 / 2004 (2ème ed.), Citoyennes Tricoteuses. Les femmes du peuple à Paris pendant la Révolution Française, Aix-en-Provence, Alinéa / Paris, Perrrin.

- 2003, Les Femmes dans la société française. XVIe-XVIIIe siècle, Paris, Armand Colin.

- 1996, «Le vote des femmes pendant la Révolution française », in Éliane Viennot (dir.), La Démocratie 'à la française' ou les femmes indésirables, Paris, Publications de l'Université Paris 7, pp. 199-211.

Houbre Gabrielle, 2003, Histoire de la grandeur et de la décadence de Marie Isabelle, modiste, dresseuse de chevaux, femme d'affaires, etc., Paris, Perrin.

Marand-Fouquet Catherine, 1989, La Femme au temps de la Révolution, Paris, Stock.

Steinberg Sylvie, 2001, La Confusion des sexes. Le travestissement de la Renaissance à la Révolution, Paris, Fayard.

- 1999, « Un brave cavalier dans la guerre de Sept Ans, Marguerite dite Jean Goubler », Clio H.F.S , $\mathrm{n}^{\circ} 10$ « Femmes travesties : un 'mauvais' genre », pp. 145-154.

Viennot Éliane, 1997, « Les femmes dans les troubles du XVI ${ }^{\mathrm{e}}$ siècle », Clio H.F.S, n 5 « Guerres civiles », pp. 79-96.

Villiers Marc de, 1910, Histoire des clubs de femmes et des légions d'Amazones, Paris.

Vovelle Michel, 1986, La Révolution française. Images et récit, Paris, Messidor/Livre Club Diderot, 5 vol.

\section{NOTES}

1. La Femme a le mousquet, l'époux la quenouille, Paris, Musée Carnavalet, reproduite dans Duby Perrot 1991 : III, 231.

2. Guette $1982: 150,58,173$. Cf. aussi 103, 115, 116.

3. Viennot 1997 et Steinberg 2001. Cf. également Cuénin 1987 et Godineau 2003.

4. De Vernon, L'Amazone chrétienne, 1678, cité par Cuénin 1992 et Steinberg 2001. $\mathrm{M}^{\mathrm{me}}$ de la Guette raconte avec fierté que des soldats lorrains cantonnant près de chez elle l'appelaient « la SaintBalmont de la Brie » (Guette $1982: 103$ ).

5. $\mathrm{M}^{\text {me }}$ de Saint-Balmont est «la merveille de son temps et pour sa valeur et pour sa belle conduite ", écrit $\mathrm{M}^{\text {me }}$ de la Guette (Guette 1982 : 103).

6. Tavannes, Mémoires du capitaine Gaspard de Saulx-Tavannes, cité par Steinberg 2001: 219 (souligné par nous).

7. Steinberg, $2001: 226-238$.

8. Steinberg, $2001: 275-285$.

9. Ordonnances de 1687, 1727, 1768 (Steinberg 1999 : 147). 
10. Moscou, Musée Pouchkine. Reproduit dans Farge 1996 : 40-41, à partir d'une gravure de la Bibliothèque de l'Arsenal.

11. Corvisier 1964 ; Steinberg 1999 et 2001. Étant donnés les problèmes de conservation des archives, la majorité des cas date du XVIII ${ }^{\mathrm{e}}$ siècle.

12. Lettre du commissaire des guerres qui demande une pension pour elle, citée par Steinberg $2001: 59$.

13. Créées au Moyen Âge, les milices bourgeoises étaient formées par les habitants d'une ville qui y détenaient le droit de bourgeoisie. Destinées à assurer la défense de la cité et le maintien de l'ordre public, elles furent vidées de l'essentiel de leurs pouvoirs par la monarchie, notamment après la Fronde. Les femmes n'en faisaient pas partie, mais la veuve d'un «bourgeois » devait souvent fournir un homme ou une somme d'argent en remplacement de son défunt mari.

14. Godineau 1988 et 1996.

15. Applewhite Lévy 1990.

16. Villiers 1910.

17. A.N., AEII 1252 CI 90; Archives Parlementaire (A.P.), t. 39, p. 423 . Née en 1768 de parents marchands de chocolat, Pauline Léon avait déjà une vie révolutionnaire bien remplie : présente dans la rue le 14 juillet 1789, et au Champ-de-Mars le 17 juillet 1791 pour signer la pétition réclamant la déchéance du roi, elle fréquentait le club des Cordeliers, la Société Fraternelle des Patriotes des Deux Sexes, la société fraternelle (mixte) de la section du Luxembourg et avait signé "un grand nombre de pétitions patriotiques». Parmi les signataires (domestiques, épouses d'artisans, couturières, marchandes détaillantes), se trouvaient plusieurs membres de sociétés populaires et des femmes qui poursuivirent pendant plusieurs années leurs activités politiques (Godineau 1988).

18. Publié dans Les Femmes... 1982 : II, $\mathrm{n}^{\circ} 40$.

19. A.N., C154 d. 292bis.

20. A.N., F7 4774/9.

21. Journal des débats et de la correspondance de la société des Jacobins, 14 mai 1793, n 412.

22. Godineau 1988 : 129-163.

23. La force de la sans-culotterie féminine pendant l'été 1793 avait donné une importance accrue aux aspirations à l'égalité politique. Sur la cocarde et sur ce contexte : Godineau 1988 : 143-179.

24. A.P., t. 78 p. 21. Moins précis et moins fiable, le compte rendu du Moniteur rapporte qu'il les aurait traitées d'aventurières et de chevalières errantes. La veille, des dames de la Halle avaient violemment attaqué les Républicaines Révolutionnaires, accusées d'être armées et de vouloir forcer toutes les femmes à porter bonnet rouge et pantalons. Après le discours de Fabre, une des marchandes venues en députation à la Convention demandait « l'abolition de toutes les Sociétés de femmes ".

25. A.N., DXLII $n^{\circ} 11$, rapport du 8 brumaire an II.

26. Godineau 1988.

27. B.N.F. Estampes, reproduit dans Vovelle 1986 : III, 241.

28. Lettre au ministre de la Guerre et rapport de Carnot, cités par Bertaud $1983: 157$.

29. A.N., C 295 d. 993.

30. A.N., F7 4704, W 271 d. Miranda et Journal des Débats..., n 412, 14 mai 1793.

31. Cité par Bertaud $1983: 160$.

32. Chassin Hennet 1899; Gerbaux 1904; Brice 1913 ; Bertaud 1979 et 1983 ; Godineau 1988; Steinberg 2001 ; Day-Hickman 2004 (approche historio-graphique).

33. Par exemple, au cabinet des Estampes de la B.N.F.: Poisson, Amazone nationale; Leyrac et Barrau son épouse, tous deux grenadiers; General Comendantin der Sansculottes... (caricature allemande).

34. B.N.F., Lb41/2791. Le texte est suivi d'une chanson du citoyen Poirier, appelant les femmes à marcher aux frontières. Cf. Godineau 1988 : 239-240. 
35. Du nom des deux célèbres sœurs Félicité et Théophile Fernig (22 et 17 ans), aides de camp de Dumouriez.

36. Par exemple: Nouvelle proclamation pour enrôler trois cent mille filles ou femmes pour aller aux frontières, qui appelle ces « chasseresses » à " voltiger sur les bords de Cythère ", cité par Villiers $1910: 87$.

37. Bertaud 1983: 154 (M. Charpentier) ; A.P., t. 67 p. 502-503 (Pochetat); A.N., F7 4652 d. Communeau. Les citoyennes Charpentier et Communeau servaient probablement dans la même division de gendarmes, celle des célèbres Vainqueurs de la Bastille. Le bataillon de Saint-Denis (Paris) accueillait Catherine Pochetat et une autre combattante, Félicité Duquet, dite Vadeboncœur.

38. Adresse en sa faveur du général de division Schavenburg au ministre de la Guerre : A.P., t. 73 p. 86.

39. Pétitions lues à la Convention les 30 nivôse et 5 ventôse an II : A.N., C289, C295 d. 985 p. 28.

40. A.N., F7 4652 ; Gerbaux 1904 : 57.

41. Lorsque fut découverte son identité, la femme Favre échappa au massacre des autres prisonniers (A.N., F7 4704), tandis que Madeleine Petitjean, femme Reversy (49 ans), subit de la part de «nos barbares ennemis » «tout ce que la férocité peut inspirer de plus cruel» (cité par Gerbaux 1904 : 57).

42. Archives de la Préfecture de Police (A.P.P.), AA 140, f. 105.

43. Moniteur : XVII, 739 (séance des Jacobins du 21 septembre).

44. Moniteur : XVI, 270 (décret du 30 avril) et XVIII, 656 (Romme le 22 frimaire an II) ; A.N., W 78 pl. $4 \mathrm{n}^{\circ} 247$ (lettre d'un lieutenant du 7 ventôse an II).

45. Qui aurait peut-être touché 20 à 30\% des effectifs (Bertaud 1983 : 167).

46. Godineau 2003 : 162-168.

47. Avant même le décret, quelques-unes avaient été renvoyées, ou avaient elles-mêmes demandé à se retirer, après avoir été reconnues femmes.

48. Non sans regrets, semble-t-il, pour le sous-lieutenant Pochetat: le Comité de la Guerre déclare le 26 juin 1793 que, s'il avait pu, il aurait fait une exception en sa faveur car: "la Convention, en éloignant les femmes des armées, n'a vraisemblablement point entendu enchaîner le zèle et les efforts magnanimes de celles qui combattent glorieusement pour la République. Cependant le décret est porté... » (A.P., t. 67, p. 503).

49. A.N., F7 4702.

50. Cité par Chassin Hennet $1899: 168$.

51. Bertaud 1979 et 1983. Houbre 2003.

52. A.N., C 255 d. 479 et Chassin Hennet 1899 : 402.

53. Bertaud $1983: 165-166$.

54. J'ai pu calculer le temps passé à l'armée par vingt-neuf d'entre elles : huit ont servi moins d'un an, treize de un à trois ans et huit plus de trois ans.

55. Séance du 8 ventôse an II des Jacobins, Moniteur : XIX, 590.

56. Les Révolutions de Paris, 5-12 janvier 1793, $\mathrm{n}^{\circ} 183$.

57. Recueil... an II : IV n 23 ; I, 23-24; V, 8-9.

58. La Rochejaquelein, Mémoires, cités par Steinberg $2001: 76,239$.

59. Journal des Débats ... $\mathrm{n}^{\circ}$ 412. À Paris, tous les hommes devaient être pourvus d'une carte de citoyen (ou carte de sûreté) délivrée par leur section, et cela qu'ils jouissent ou non des droits politiques. En revanche, les femmes n'en possédaient pas.

60. Cf. par exemple la conception rousseauiste du rapport amoureux, construit par l'attaque (masculine) et la défense (féminine) : Rousseau 1966 : 466-469.

61. Moniteur : XVI, 539.

62. Cité par Bertaud $1983: 151$. 


\section{RÉSUMÉS}

Alors que la guerre était associée au masculin, quelques femmes en France ont combattu au cours des guerres civiles des $\mathrm{XVI}^{\mathrm{e}}-\mathrm{XVII}{ }^{\mathrm{e}}$ siècles ou ont servi dans les armées royales, qu'accompagnaient de plus de nombreuses civiles. Avec la Révolution française, le service dans la garde nationale et, moins directement, dans l'armée est lié à la citoyenneté. En réclamant le port des armes au sein de la garde nationale, des militantes révolutionnaires revendiquaient ainsi un des droits politiques du citoyen, ce qui provoqua un ferme refus. Mues par le désir de défendre la République et de partager avec les hommes la gloire de se battre pour elle, des citoyennes s'engagèrent par ailleurs individuellement dans les armées, où elles continuèrent à servir après le décret du 30 avril 1793 qui les en chassait. Les demandes d'armement des militantes et les réponses qui leur sont apportées, ainsi que les histoires, les propos des soldates et les réactions qu'elles ont suscitées permettent d'analyser l'association entre masculin, armes et citoyenneté et d'étudier les mécanismes de l'exclusion des femmes des armées.

Although war is considered a masculine activity, some French women fought in the civil wars of the 16th and 17th centuries or served in the royal armies, which many women accompanied as civilians. During the French Revolution, service within the National guard and, less directly, in the army was linked to citizenship. As a result, the militant revolutionary women who demanded the right to bear arms in the National guard were claiming one of the political rights of citizenship, and thus experienced a firm rejection of their demand. In their desire to defend the Republic and to share with men the glory of fighting for it, citoyennes enrolled individually in the army and continued to serve even after the decree of 30 April 1793 banned them from serving. Women's requests to be armed, the responses these generated, as well as the stories, the remarks of women soldiers, and the reactions they inspired offer the means to analyze the relationship between masculinity, arms, and citizenship, as well as to study the mechanisms that excluded women from the army.

\section{INDEX}

Index chronologique : Ancien régime, Révolution française

Index géographique : France

Mots-clés : armée, citoyenneté, femmes, garde nationale, guerrière

\section{AUTEUR}

\section{DOMINIQUE GODINEAU}

Dominique Godineau est maîtresse de conférences d'histoire moderne à l'Université Rennes 2, chercheuse au CRHISCO et membre du comité de rédaction de CLIO. Spécialiste de l'histoire de la Révolution française et de l'histoire des femmes à l'époque moderne, elle a publié de nombreux articles sur ces thèmes ainsi que Citoyennes tricoteuses. Les femmes du peuple à Paris pendant la Révolution française (Alinéa, 1988 ; rééd. : Perrin, 2004) et Les Femmes dans la société française. $16^{e}-18^{e}$ siècle (Armand Colin, 2003). Elle a co-dirigé (avec Luc Capdevila et alii) Le Genre face aux mutations. Masculin et féminin, du Moyen Âge à nos jours (PUR, 2003). Elle travaille actuellement sur le suicide au XVIII siècle. 